\title{
THE CHARACTERISTICS OF ASYMPTOTIC OSCULATING QUADRICS OF A CURVE ON A SURFACE
}

\author{
BUCHIN SU ${ }^{1}$
}

1. Introduction. Lane ${ }^{2}$ has derived the equations of asymptotic osculating quadrics of a curve $C$ on a surface $S$, employing Wilczynski's notation and deducing some of their fundamental properties. We shall now investigate the characteristics of these quadrics along $C$. For the sake of convenience the normal tetrahedron of $\mathrm{Cartan}^{3}$ is utilized throughout this note. In terms of the local coordinates with respect to this tetrahedron we have expressed simply the equations of asymptotic osculating quadrics and their characteristics and therefrom obtained new configurations projectively connected with a surface.

2. The normal tetrahedron of Cartan. Let us consider the directrices of Wilczynski at a generic point $M$ of a non-ruled surface $S$; the first of them intersects the quadric of Lie at another point $M_{3}$ and the second meets the asymptotic tangents at the points $M_{1}$ and $M_{2}$. The tetrahedron $\left\{M M_{1} M_{2} M_{3}\right\}$ is known as the normal tetrahedron of Cartan. If we denote, for simplicity, the corresponding projective coordinates of these points $M, M_{1}, M_{2}, M_{3}$ by the same notations, then they are solutions of the following system of differential equations: ${ }^{4}$

$$
\begin{aligned}
& \frac{\partial M}{\partial u}=\frac{1}{2} M \frac{\partial}{\partial u} \log \gamma+M_{1}, \\
& \frac{\partial M_{1}}{\partial u}=B^{2} M-\frac{1}{2} M_{1} \frac{\partial}{\partial u} \log \gamma+\beta M_{2}, \\
& \frac{\partial M_{2}}{\partial u}=K M+\frac{1}{2} M_{2} \frac{\partial}{\partial u} \log \gamma+M_{3}, \\
& \frac{\partial M_{3}}{\partial u}=A^{2} \beta M+K M_{1}+B^{2} M_{2}-\frac{1}{2} M_{3} \frac{\partial}{\partial u} \log \gamma ;
\end{aligned}
$$

Received by the editors April 24, 1943.

1 Research Professor in Mathematics, Academia Sinica.

2 E. P. Lane, The asymptotic osculating quadrics of a curve on a surface, Bull. Amer. Math. Soc. vol. 33 (1927) pp. 195-200.

${ }^{3} \mathrm{Cf}$., for example, Fubini and Čech, Introduction a la géométrie projective différentielle des surfaces, Paris, 1931, p. 236.

4 These were first used in this form by S. Finikoff, Sur les couples de surfaces dont les asymptotiques se correspondent et qui, aux points correspondants, ont les mêmes directrices de Wilczynski, C. R. Acad. Sci. Paris vol. 197 (1933) pp. 883-885. 
(1)

$$
\begin{aligned}
& \frac{\partial M}{\partial v}=\frac{1}{2} M \frac{\partial}{\partial v} \log \beta+M_{2}, \\
& \frac{\partial M_{1}}{\partial v}=\bar{K} M+\frac{1}{2} M_{1} \frac{\partial}{\partial v} \log \beta+M_{3}, \\
& \frac{\partial M_{2}}{\partial v}=A^{2} M+\gamma M_{1}-\frac{1}{2} M_{2} \frac{\partial}{\partial v} \log \beta, \\
& \frac{\partial M_{3}}{\partial v}=B^{2} \gamma M+A^{2} M_{1}+\bar{K} M_{2}-\frac{1}{2} M_{3} \frac{\partial}{\partial v} \log \beta,
\end{aligned}
$$

where the components of displacements of the tetrahedron $\beta, \gamma, A, B$, $K, \bar{K}$ satisfy the integrability conditions

$$
\begin{aligned}
& 2 K=\beta \gamma-\frac{\partial^{2}}{\partial u \partial v} \log \beta, \quad 2 \bar{K}=\beta \gamma-\frac{\partial^{2}}{\partial u \partial v} \log \gamma, \\
& \frac{\partial\left(A^{2}\right)}{\partial u}=K \frac{\partial}{\partial v} \log (K \beta), \quad \frac{\partial\left(B^{2}\right)}{\partial v}=\bar{K} \frac{\partial}{\partial u} \log (\bar{K} \gamma), \\
& A \frac{\partial(A \beta)}{\partial v}=B \frac{\partial(B \gamma)}{\partial u} \text {. }
\end{aligned}
$$

Any point $P$ in space can now be represented by the local coordinates $\left(y_{1}, y_{2}, y_{3}, y_{4}\right)$ with respect to the tetrahedron $\left\{M M_{1} M_{2} M_{3}\right\}$,

$$
P=y_{1} M+y_{2} M_{1}+y_{3} M_{2}+y_{4} M_{3} \text {. }
$$

From (1) we easily show that the conditions of immovability for $P$ are

$$
\begin{aligned}
& \frac{\partial y_{1}}{\partial u}=-\frac{1}{2}\left(\frac{\partial}{\partial u} \log \gamma\right) y_{1}-B^{2} y_{2} \quad-K y_{3} \quad-A^{2} \beta y_{4}, \\
& \frac{\partial y_{2}}{\partial u}=\quad-y_{1}+\frac{1}{2}\left(\frac{\partial}{\partial u} \log \gamma\right) y_{2} \quad * \quad-K y_{4}, \\
& \frac{\partial y_{3}}{\partial u}=* \quad-\beta y_{2} \quad-\frac{1}{2}\left(\frac{\partial}{\partial u} \log \gamma\right) y_{3}-B^{2} y_{4}, \\
& \text { (4) } \\
& \frac{\partial y_{4}}{\partial u}=* \quad * \quad-y_{3} \quad+\frac{1}{2}\left(\frac{\partial}{\partial u} \log \gamma\right) y_{4} ; \\
& \frac{\partial y_{1}}{\partial v}=-\frac{1}{2}\left(\frac{\partial}{\partial v} \log \beta\right) y_{1}-\bar{K} y_{2} \quad-A^{2} y_{3} \quad-B^{2} \gamma y_{4}, \\
& \frac{\partial y_{2}}{\partial v}=* \quad-\frac{1}{2}\left(\frac{\partial}{\partial v} \log \beta\right) y_{2}-\gamma y_{3} \quad-A^{2} y_{4}, \\
& \frac{\partial y_{3}}{\partial v}=-y_{1} \quad * \quad+\frac{1}{2}\left(\frac{\partial}{\partial v} \log \beta\right) y_{3}-\bar{K} y_{4},
\end{aligned}
$$

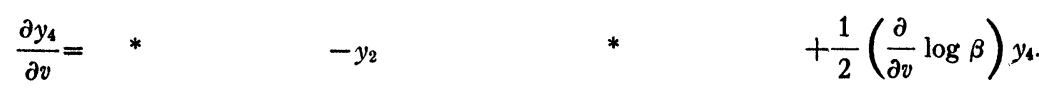


3. Equations. Suppose that a curve $C$ on $S$ is given by the equation

$$
u=u(v) \text {. }
$$

The asymptotic ruled surface $R_{u}$ generated by the asymptotic $u$ tangents of $S$ along $C$ is given by

$$
P=M+\rho M_{1},
$$

where $\rho$ and $v$ are independent parameters, the variable $u$ in $M$ and $M_{1}$ being given by (5).

In virtue of (1) there is no difficulty in calculating the partial derivatives of $P$ with respect to $\rho$ and $v$. Denoting $d u / d v, d^{2} u / d v^{2}, d^{3} u / d v^{3}$ by $u^{\prime}, u^{\prime \prime}, u^{\prime \prime \prime}$ respectively we have

$$
\begin{aligned}
P_{\rho}= & M_{1} \\
P_{v}= & \left\{\frac{1}{2} u^{\prime} \frac{\partial}{\partial u} \log \gamma+\frac{1}{2} \frac{\partial}{\partial v} \log \beta+\rho\left(B^{2} u^{\prime}+\bar{K}\right)\right\} M \\
& +\left\{u^{\prime}+\frac{1}{2} \rho\left(-u^{\prime} \frac{\partial}{\partial u} \log \gamma+\frac{\partial}{\partial v} \log \beta\right)\right\} M_{1} \\
& +\left(1+\rho \beta u^{\prime}\right) M_{2}+\rho M_{3}, \\
P_{v \rho}= & \left(B^{2} u^{\prime}+\bar{K}\right) M+\frac{1}{2}\left(-u^{\prime} \frac{\partial}{\partial u} \log \gamma+\frac{\partial}{\partial v} \log \beta\right) M_{1} \\
& +\beta u^{\prime} M_{2}+M_{3}, \\
P_{v v}= & (*) M+\left(^{*}\right) M_{1} \\
& +\left\{u^{\prime} \frac{\partial}{\partial u} \log \gamma+\beta u^{\prime 2}+\rho\left(2 B^{2} u^{\prime}+2 \bar{K}\right.\right. \\
& +2\left\{u^{\prime}+\rho\left(-u^{\prime} \frac{\partial}{\partial u} \log \gamma+\beta u^{\prime 2}\right)\right\} M_{3},
\end{aligned}
$$

(7)

so that the differential equation of the curved asymptotics of $R_{u}$,

$$
2\left(P P_{\rho} P_{v} P_{v \rho}\right) d \rho+\left(P P_{\rho} P_{v} P_{v v}\right) d v=0,
$$

becomes

$$
\frac{d \rho}{d v}=a \rho^{2}+b \rho-u^{\prime}
$$

\footnotetext{
6 The parenthesis denotes a determinant of the four points there inclosed.
} 
where we have placed

$$
\begin{aligned}
a= & \bar{K}+B^{2} u^{\prime}+\frac{1}{2} \beta u^{\prime \prime}+\frac{1}{2} \beta u^{\prime 2} \frac{\partial}{\partial u} \log (\beta \gamma) \\
& +\frac{1}{2} \beta u^{\prime} \frac{\partial}{\partial v} \log \beta-\frac{1}{2} \beta^{2} u^{\prime 3}, \\
b= & u^{\prime} \frac{\partial}{\partial u} \log \gamma-\beta u^{\prime 2} .
\end{aligned}
$$

The osculating quadric $Q_{u}$ or $R_{u}$ along its generator $M M_{1}$ is the asymptotic osculating quadric of one family, and a second asymptotic osculating quadric $Q_{v}$ of $C$ at $M$ is obtained by using the other family of asymptotics of $S$. It is clear, therefore, that a generic point $Z$ on $Q_{u}$ must be represented by

$$
Z=P+\sigma \frac{d P}{d v}=M+\rho M_{1}+\sigma\left(P_{v}+P_{\rho} \frac{d \rho}{d v}\right),
$$

$\sigma$ being another parameter.

Substitution of (7) and (8) in (10) gives the local coordinates of the point $Z$, namely,

$$
\begin{aligned}
y_{1} & =1+\sigma\left\{\frac{1}{2} u^{\prime} \frac{\partial}{\partial u} \log \gamma+\frac{1}{2} \frac{\partial}{\partial v} \log \beta+\rho\left(B^{2} u^{\prime}+\bar{K}\right)\right\}, \\
\text { (11) } y_{2} & =\rho+\rho \sigma\left\{\frac{1}{2} u^{\prime} \frac{\partial}{\partial v} \log \gamma+\frac{1}{2} \frac{\partial}{\partial v} \log \beta-\beta u^{\prime 2}\right\}+a \rho^{2} \sigma, \\
y_{3} & =\left(1+\rho \beta u^{\prime}\right) \sigma, \\
y_{4} & =\rho \sigma .
\end{aligned}
$$

Thus we obtain the equation of the first asymptotic osculating quadric $Q_{u}$ of $C$ at $M$ :

$$
\begin{aligned}
& y_{1} y_{4}-y_{2} y_{3}+\beta u^{\prime} y_{2} y_{4}-\beta u^{\prime 2} y_{3} y_{4} \\
& \quad+\frac{1}{2} \beta\left\{u^{\prime \prime}+\beta u^{\prime 3}+u^{\prime 2} \frac{\partial}{\partial u} \log (\beta \gamma)+u^{\prime} \frac{\partial}{\partial v} \log \beta\right\} y_{4}^{2}=0 .
\end{aligned}
$$

The equation of the second asymptotic osculating quadric $Q_{v}$ of $C$ at $M$ is

$$
\begin{aligned}
& u^{\prime 3}\left(y_{1} y_{4}-y_{2} y_{3}\right)-\gamma u^{\prime} y_{2} y_{4}+\gamma u^{\prime 2} y_{3} y_{4} \\
& \quad+\frac{1}{2} \gamma\left\{-u^{\prime \prime}+\gamma+u^{\prime} \frac{\partial}{\partial v} \log (\beta \gamma)+u^{\prime 2} \frac{\partial}{\partial u} \log \gamma\right\} y_{4}^{2}=0 .
\end{aligned}
$$


4. Characteristics. The asymptotic osculating quadrics $Q_{u}$ (or $Q_{v}$ ) at $M$ and its consecutive point $M^{\prime}$ on the curve $C$ intersect each other at the asymptotic tangent $u$ (or $v$ ) and two other lines, which are called the characteristic of $Q_{u}$ (or $Q_{v}$ ) along $C$. In order to derive the equations of the characteristics of $Q_{u}$ we have to set the derivative along $C$ of the left-hand member of (12) equal to zero, remembering that the derivatives of $y$ 's should be determined by (4). A simple calculation, which we shall omit here, suffices to demonstrate that the equations of the characteristic in consideration are (12) and

$$
C_{33} y_{3}^{2}-C_{34} y_{3} y_{4}+C_{44} y_{4}^{2}=0,
$$

where we have placed

$$
\begin{aligned}
C_{33}= & \beta u^{\prime 3}+\gamma, \\
C_{34}= & \beta u^{\prime}\left\{3 u^{\prime \prime}+\beta u^{\prime 3}+\left(\frac{\partial}{\partial u} \log \left(\beta^{2} \gamma\right)\right) u^{\prime 2}\right. \\
& \left.+3\left(\frac{\partial}{\partial v} \log \beta\right) u^{\prime}+\gamma\right\}, \\
C_{44}= & -A^{2} \beta u^{\prime}-B^{2} \gamma+\beta u^{\prime 2}\left(B^{2} u+\bar{K}\right)-\beta u^{\prime}\left(K u^{\prime}+A^{2}\right) \\
& +\frac{1}{2} \beta\left\{u^{\prime \prime}+\beta u^{\prime 3}+\left(\frac{\partial}{\partial u} \log (\beta \gamma)\right) u^{\prime 2}\right. \\
& \left.+\left(\frac{\partial}{\partial u} \log \beta\right) u^{\prime}\right\}\left\{u^{\prime} \frac{\partial}{\partial u} \log \gamma+\frac{\partial}{\partial v} \log \beta\right\} \\
& +\frac{1}{2} \beta\left\{u^{\prime \prime \prime}+\left(\frac{\partial}{\partial u} \log \left(\beta^{3} \gamma^{2}\right)\right) u^{\prime} u^{\prime \prime}\right. \\
& +2\left(\frac{\partial}{\partial v} \log \beta\right) u^{\prime \prime}+3 \beta^{2} u^{\prime 2} u^{\prime \prime} \\
& +2\left(\frac{\partial}{\partial u} \log \beta\right) u^{\prime 4}+\left(2 \frac{\partial}{\partial v} \log \beta+\frac{\partial^{2}}{\partial u^{2}} \log (\beta \gamma)\right. \\
& \left.+\frac{\partial}{\partial u} \log (\beta \gamma) \frac{\partial}{\partial u} \log \beta\right) u^{\prime 3} \\
& +\left(\frac{\partial^{2}}{\partial u \partial v} \log \left(\beta^{2} \gamma\right)+2 \frac{\partial}{\partial u} \log (\beta \gamma) \frac{\partial}{\partial v} \log \beta\right) u^{\prime 2} \\
& \left.+\left(\frac{\partial^{2}}{\partial v^{2}} \log \beta+\left(\frac{\partial}{\partial v} \log \beta\right)^{2}\right) u^{\prime}\right\} .
\end{aligned}
$$


From (14) it follows that the characteristic of $Q_{u}$ along $C$ consists of two straight lines intersecting the asymptotic $u$-tangent and the latter counted twice.

In a similar way we obtain another pair of lines intersecting the asymptotic tangent $M M_{2}$ as the second quadric moves along $C$. These four lines furnish obviously a generalization of the well known quadrilateral of Demoulin.

By means of (14) we can deduce some remarkable results. ${ }^{6}$

(I) If the asymptotic tangent $M M_{2}$ is a part of the characteristic of the asymptotic osculating quadric $Q_{u}$ of $C$ at $M$, then $C$ must be tangent to a Darboux curve at $M$, and conversely.

The necessary and sufficient condition for this is $C_{33}=0$, namely,

$$
\beta u^{\prime 3}+\gamma=0,
$$

which represents the Darboux directions of the surface at $M$.

(II) In order that the characteristic of the first and second asymptotic osculating quadrics along every Darboux curve of a surface $S$ should decompose into two asymptotic tangents, each being counted twice, the necessary and sufficient condition is that $S$ be a surface of coincidence.

In fact, we have $C_{33}=0, C_{34}=0$, so that (14) becomes $y_{4}^{2}=0$. In consequence, the characteristic is given by the equations

$$
y_{4}^{2}=0, \quad y_{2} y_{3}=0 \text {. }
$$

For a Darboux curve we have

(19) $\beta u^{\prime 3}+\gamma=0, \quad u^{\prime \prime}=-\frac{1}{3} u^{\prime 2} \frac{\partial}{\partial u} \log (\gamma / \beta)-\frac{1}{3} u^{\prime} \frac{\partial}{\partial v} \log (\gamma / \beta)$.

Substituting these in the condition $C_{34}=0$, namely,

$$
3 u^{\prime \prime}+\beta u^{\prime 3}+u^{\prime 2} \frac{\partial}{\partial u} \log \left(\beta^{2} \gamma\right)+3 u^{\prime} \frac{\partial}{\partial v} \log \beta+\gamma=0,
$$

and taking account of the fact that this equation must hold for the three Darboux curves, we arrive at

$$
\frac{\partial}{\partial v} \log \beta=0, \quad \frac{\partial}{\partial v} \log \gamma=0,
$$

that is, both $\beta$ and $\gamma$ are functions of $u$ alone.

${ }^{6}$ Cf. S. C. Chang, On the surfaces of coincidence, Bull. Amer. Math. Soc. vol. 49 (1943) pp. 900-903. 
Since the same condition also holds for the second asymptotic osculating quadrics, we have that $\beta$ and $\gamma$ must be functions of $v$ alone, and therefore that they are constants. Hence the surface in question is a surface of coincidence.

(III) If the characteristics of the quadrics $Q_{u}$ and $Q_{v}$ along every Darboux curve of a surface $S$ are indeterminate, that is, the asymptotic osculating quadrics along every Darboux curve are stationary, then $S$ must be projectively equivalent to the cubic surface $x y z=1$.

For we have in this case

$$
C_{33}=0, \quad C_{34}=0, \quad C_{44}=0 .
$$

We may put $\beta=\gamma=1$, which gives

$$
A^{2}=0, \quad B^{2}=0 .
$$

The equation to the surface may easily be found by integrating the system (1).

5. Associate directrices. We come now to demonstrate that a certain pair of covariant lines can be constructed by means of the asymptotic osculating quadrics. Consider, for instance, the first quadric $Q_{u}$ given by (12). As was shown before, there exists an element of the second order, $E_{2}$, corresponding to each Darboux tangent, such that the characteristic of $Q_{u}$ at the point $M$ decomposes into the two asymptotic tangents $M M_{1}$ and $M M_{2}$, each being counted twice. We have therefore a plane containing this $E_{2}$. In order to find the equation of this plane we merely have to substitute the value of $u^{\prime \prime}$ given by (20) in the equation of the osculating plane of $C$ at $M$ :

$$
\begin{aligned}
& 2 u^{\prime}\left(x-u^{\prime} y\right) \\
& -\left(u^{\prime \prime}+u^{\prime 2} \frac{\partial}{\partial u} \log (\beta \gamma)+\gamma-u^{\prime} \frac{\partial}{\partial v} \log (\beta \gamma)-\beta u^{\prime 3}\right) z=0,
\end{aligned}
$$

where $x, y, z$ denote the nonhomogeneneous coordinates of a point with respect to the tetrahedron of Fubini at $M$. The result of carrying out the computation is

$$
2 u^{\prime}\left(x-u^{\prime} y\right)-\left\{\phi u^{\prime 2} / 3-\psi u^{\prime}+2 \gamma\right\} z=0,
$$

where

$$
\begin{gathered}
u^{\prime}=-\epsilon(\gamma / \beta)^{1 / 3} \quad\left(\epsilon \neq 1, \epsilon^{3}=1\right), \\
\phi=\frac{\partial}{\partial u} \log \left(\beta \gamma^{2}\right), \quad \psi=\frac{\partial}{\partial v} \log \left(\beta^{2} \gamma\right) .
\end{gathered}
$$


The three planes (22) corresponding to the Darboux tangents at $M$ form a trihedron, and the polar of the tangent plane of the surface at $M$ with respect to this is

$$
x+\psi z / 2=0, \quad y+\phi z / 6=0 .
$$

If the second quadric $Q_{v}$ is used instead of $Q_{u}$, a second covariant line is found to be

$$
x+\psi z / 6=0, \quad y+\phi z / 2=0 .
$$

These lines are analogous to the directrices of Sullivan ${ }^{7}$ and will be called the associate directrices of the surface at $M$. The first of them, given by (25), is the intersection of the plane containing the asymptotic $v$-tangent and the first directrix of Wilczynski and the plane containing the asymptotic $u$-tangent and the first principal ray $c(-1 / 6)$ of Fubini. A similar construction is obtained for the second associate directrix. It shall be noted that the asymptotic $u$-tangent, the first directrix of Sullivan, the first associate directrix and the first directrix of Wilczynski are coplanar and the double ratio of them in this order is equal to -3 .

There are two planes through each Darboux tangent at $M$, one being (22) and the other for $Q_{v}$ :

$$
x-u^{\prime} y+(1 / 2)\left\{\psi-\phi u^{\prime} / 3+2 \beta u^{\prime 2}\right\} z=0 .
$$

The harmonic conjugate of the tangent plane of the surface with respect to them osculates the corresponding Darboux curve at $M$, as we can easily show from the equations (19) and (21).

6. Associate cones. As was shown by Lane, ${ }^{8}$ the directrices of Sullivan are related to a certain pair of cones of the third class enveloped by the osculating planes at $M$ of the extremals of certain integrals. We shall here prove that analogous cones also exist in the case of associate directrices.

Let us consider again the characteristic of the first quadric $Q_{u}$ along a curve $C$ on the surface $S$. For a given non-Darboux direction equation (14) shows that we can always adjust an element of the second order, $E_{2}$, to the curve $C$ of the given direction at $M$, such that the planes through the asymptotic $u$-tangent and the characteristic lines are harmonic conjugate to the tangent plane and the plane through the first directrix of Wilczynski. The plane containing this $E_{2}$ cor-

${ }^{7}$ C. T. Sullivan, Scroll directrix curves, Trans. Amer. Math. Soc. vol. 16 (1915) pp. 199-214.

${ }^{8}$ E. P. Lane, Le direttrici di Sullivan, Bollettino della Unione Matematica Italiana vol. 5 (1926) pp. 214-215. 
responding to every non-Darboux direction at $M$ envelopes a cone of the third class

$$
\gamma u_{1}^{3}+2 \beta u_{2}^{3}+3\left(\psi u_{1} / 2+\phi u_{2} / 6-u_{3}\right) u_{1} u_{2}=0,
$$

where $u_{1}, u_{2}, u_{3}$ denote the coordinates of a plane through $M$. The three cuspidal planes of this cone evidently pass through the first associate directrix.

In a similar way a second cone of the third class

$$
2 \gamma u_{1}^{3}+\beta u_{2}^{3}+3\left(\psi u_{1} / 6+\phi u_{2} / 2-u_{3}\right) u_{1} u_{2}=0
$$

is obtained.

In virtue of these cones we can further construct a third cone of the third class and a new canonical ray at the point $M$ of the surface.

For this purpose, we draw the tangent planes of the cones (28) and (29) through a non-Darboux tangent at $M$ and construct the harmonic conjugate of the tangent plane of the surface with respect to them. The equation of this plane is found to be

$$
2 u^{\prime}\left(x-u^{\prime} y\right)+\left(\beta u^{\prime 3}-\gamma+\phi u^{\prime 2} / 3-\psi u^{\prime} / 3\right) z=0 .
$$

It is easily seen that the three planes corresponding to the three Segre tangents at $M$ are concurrent in the canonical ray $c(1 / 6)$ :

$$
x-\psi z / 6=0, \quad y-\phi z / 6=0 .
$$

This line and the first principal ray of Fubini harmonically separate the canonical tangent and the projective normal of the surface at $M$. The plane (30) also envelopes a cone of the third class

$$
\gamma u_{1}^{3}+\beta u_{2}^{3}-2\left(\psi u_{1} / 6+\phi u_{2} / 6+u_{3}\right) u_{1} u_{2}=0
$$

and the three cuspidal planes are concurrent in the ray $c(1 / 6)$.

National. University of Cheriang 